\section{Alternativas para la adopción de políticas centradas en el acceso a medicamentos}

\author{
Federico Tobar, ${ }^{1}$ Ignacio Drake ${ }^{2}$ \\ y Evangelina Martich ${ }^{3}$
}

Forma de citar: Tobar F, Drake I, Martich E. Alternativas para la adopción de políticas centradas en el acceso a medicamentos. Rev Panam Salud Publica. 2012;32(6):457-63.

\begin{abstract}
SINOPSIS
América Latina está incorporando regulaciones que incidan en el costo y el gasto en medicamentos. Sus objetivos centrales son cuatro: i) garantizar la competitividad en el mercado, ii) asegurar precios asequibles a los consumidores individuales (canal comercial), iii) contener el gasto público en medicamentos (canal institucional) y iv) garantizar la eficiencia del gasto en medicamentos. La experiencia de América Latina difiere de la de los países de regiones desarrolladas. En estas últimas los países tienden a converger en sus políticas, tanto en la promoción de medicamentos genéricos y estrategias de control de precios, como en las medidas de racionalización y contención del gasto farmacéutico. En contraste, en América Latina ciertas debilidades institucionales impiden la consolidación y aplicación de una politica regulatoria efectiva. El presente trabajo revisa la experiencia acumulada en la adopción de regulaciones económicas dirigidas a reducir el gasto y mejorar el acceso a los medicamentos, postula lecciones aprendidas a nivel internacional y ofrece recomendaciones para los países de América Latina. Su propósito es aportar elementos clave para los decisores de política y las autoridades de los países interesados en llevar adelante la regulación económica de medicamentos.
\end{abstract}

Palabras clave: precio de medicamento; medicamentos genéricos; financiación de la salud; inversiones en salud; América Latina.

1 Universidad de Buenos Aires (UBA), Buenos Aires, Argentina. La correspondencia se debe dirigir a Ignacio Drake. Correo electrónico: cidrake@yahoo.com.ar

2 Ministerio de Salud de la Nación, Buenos Aires, Argentina.

3 Escola Nacional De Saúde Pública (ENSP, FIOCRUZ), Río de Janeiro, Brasil.
El acceso de la población a los medicamentos constituye uno de los desafíos sociales más importantes para los estados. Enfrentarlo con éxito implica no solo lograr la disponibilidad de medicamentos, sino también garantizar que sean usados de forma racional y se puedan adquirir a un costo asequible. En América Latina y el Caribe, a esta situación se añaden características particulares por las dificultades que tiene la población para acceder a medicamentos esenciales (1).

En este marco, promover el acceso de la gente a los medicamentos resulta un objetivo fundamental en la región, donde algunos países ya han implementado o están en proceso de implementar políticas centradas en la regulación económica de medicamentos.

A partir del relevamiento y el análisis de las experiencias de regulación de medicamentos tanto de países latinoamericanos como europeos, el presente trabajo busca aportar elementos clave para los decisores de políticas y las autoridades de los países de la región interesados en llevar adelante la regulación económica de medicamentos.

\section{EXPERIENCIAS EN LA REGULACIÓN DE MEDICAMENTOS}

Al implementar políticas para promover el acceso de la población a los medicamentos, los países no siguieron un camino único ni homogéneo (2-4). Dentro de estas políticas, las medidas de regulación económica se han expandido más lentamente que las regulaciones técnicas y parece más difícil alcanzar un consenso sobre cuál debería ser el camino más adecuado. En el cuadro 1 se presenta una taxonomía de herramientas de regulación económica que distintos países han incorporado, tanto de forma aislada como combinada. Como se puede observar, los países persiguen dos propósitos: i) controlar el costo de los medicamentos en el mercado (canal comercial) y ii) contener el gasto gubernamental en medicamentos (canal institucional). Para cada uno de ellos se identifican objetivos y herramientas posibles.

\section{Costo de los medicamentos}

Es posible identificar dos grandes grupos de estrategias regulatorias para controlar el precio de los medicamentos en el canal comercial. Unas son las estrategias procompetitivas, que esperan que la reducción de precios resulte de una mayor competencia entre los oferentes $\mathrm{y}$, por ende, no se interviene en forma directa sobre los precios; en el otro grupo están las estrategias intervencionistas, que directamente controlan el precio de venta en el canal comercial. 
CUADRO 1. Opciones para la regulación económica de medicamentos

\begin{tabular}{|c|c|c|}
\hline Propósito & Objetivo de política & Herramientas posibles \\
\hline \multirow[t]{2}{*}{$\begin{array}{l}\text { Control de costo de } \\
\text { medicamentos }\end{array}$} & $\begin{array}{l}\text { Garantizar la } \\
\text { competitividad en el } \\
\text { mercado }\end{array}$ & $\begin{array}{l}\text { Control de prácticas } \\
\text { anticompetitivas } \\
\text { Promoción de } \\
\text { genéricos }\end{array}$ \\
\hline & $\begin{array}{l}\text { Garantizar precios } \\
\text { asequibles }\end{array}$ & $\begin{array}{l}\text { Control de precios } \\
\text { y márgenes de } \\
\text { comercialización } \\
\text { Negociaciones de } \\
\text { precios }\end{array}$ \\
\hline \multirow[t]{2}{*}{$\begin{array}{l}\text { Contención del gasto } \\
\text { en medicamentos }\end{array}$} & $\begin{array}{l}\text { Contener el } \\
\text { gasto público en } \\
\text { medicamentos }\end{array}$ & $\begin{array}{l}\text { Promoción del uso de } \\
\text { genéricos } \\
\text { Cambios en las } \\
\text { modalidades de } \\
\text { contratación y } \\
\text { adquisición } \\
\text { Fijación de precios } \\
\text { para rembolso } \\
\text { Copagos }\end{array}$ \\
\hline & $\begin{array}{l}\text { Asegurar la } \\
\text { eficiencia en } \\
\text { el gasto }\end{array}$ & $\begin{array}{l}\text { Evaluación económica } \\
\text { de medicamentos y } \\
\text { financiación selectiva }\end{array}$ \\
\hline
\end{tabular}

Fuente: elaboración de los autores.

Experiencias de las estrategias regulatorias procompetitivas. La promoción del uso de medicamentos genéricos demostró ser una política efectiva para reducir el gasto y mejorar el acceso a los medicamentos (5-7). En algunos casos se simplificaron los procedimientos de entrada al mercado de una mayor cantidad de productos genéricos mediante la eliminación de restricciones relacionadas con, por ejemplo, patentes vencidas o demoras en la aprobación de los registros. En otros, se introdujeron leyes que obligaron la prescripción o la sustitución de medicamentos de marca por nombres genéricos (4).

Los países que optaron por favorecer estrategias de promoción de genéricos verificaron que los medicamentos se comercialicen en el mercado nacional a precios entre un $35 \%$ y un $50 \%$ inferiores a los de los productos de referencia.

Un problema que surge con los genéricos es que no han sido recibidos con entusiasmo en el sector comercial en razón de que los márgenes de ganancia de las farmacias son proporcionales a los precios de venta de los productos. Ante esta situación, se incorporaron incentivos de venta adicionales (p. ej. Portugal) o se estableció la obligatoriedad de la sustitución por ser la alternativa más económica (p. ej. España) $(8,9)$. Otros países optaron por la sensibilización y la divulgación de información respecto de las ventajas de usar medicamentos genéricos, a través de campañas publicitarias dirigidas a pacientes, o bien mediante incentivos y recomendaciones gubernamentales, o de los seguros de salud, para los prescriptores, pero no tuvieron resultados tan determinantes como la obligatoriedad en prescriptores y dispensadores (3).
Experiencias de las estrategias regulatorias intervencionistas. En relación con la política de control de precios de medicamentos, se distingue una diversidad de instrumentos según partan de: i) el análisis farmacológico, que evalúa las ventajas terapéuticas del medicamento; ii) la evaluación económica, que compara el costo del tratamiento con el de otros productos alternativos; iii) el precio del mismo producto en otros países, y iv) el control de la formación del precio de venta al público estableciendo márgenes de comercialización ("techos" a los márgenes de ganancias de farmacias y droguerías).

Varios países de América Latina buscaron mantener regímenes regulatorios (o canales) diferenciados para los productos competitivos, oligopólicos y monopólicos. Este enfoque ya se implementó en Brasil, Colombia y Ecuador, y se está instrumentando en El Salvador. Tal camino puede resultar técnicamente más factible que analizar la estructura de costos de producción de cada producto farmacéutico, como lo hacen algunos países europeos (entre ellos Inglaterra y España). Además, el establecimiento de regímenes diferenciales —en los que se distinguen mayores y menores grados de libertad para fijar precios- resulta más viable que implementar un control de precios de todos los productos y menos inflacionario que desregular los precios en todo el mercado.

Otra alternativa de uso generalizado en la Unión Europea (excepto Alemania y Luxemburgo) fue la implementación de un sistema de precios máximos de referencia. Para su concreción es necesario contar con información precisa y explicitar la metodología, lo que genera un impacto considerable en el corto plazo - determinado por el nivel de cobertura dentro del mercado que tengan los precios de los medicamentos incluidos. Este recurso solo es efectivo en el caso de los productos monopólicos $\mathrm{u}$ oligopólicos, porque de lo contrario se corre el riesgo de establecer precios de referencia por encima de los vigentes en el mercado. Dado que con el transcurso del tiempo se han observado algunas limitaciones si solo se aplican los precios de referencia de manera aislada, conviene articular este método con otras medidas, como por ejemplo compras centralizadas, incentivos a la utilización de genéricos o evaluaciones fármaco-económicas sobre nuevas presentaciones.

Una alternativa es la creación de agencias de evaluación de tecnologías sanitarias que incorporen la perspectiva fármaco-económica, que contribuye a la racionalidad, eficiencia y sostenibilidad de la financiación del gasto farmacéutico dentro del sistema de salud. Pero esto requiere de cierto desarrollo institucional e involucra requisitos como capacidad técnica y voluntad política para respetar y sostener las recomendaciones de la agencia.

\section{Gasto en medicamentos}

Los países europeos, Australia y Canadá incorporaron de forma intensiva distintos instrumentos de con- 
tención del gasto farmacéutico (4). Uno es la selección de los medicamentos con mayor beneficio terapéutico y más costo-efectivos para ser utilizados, entre otros, en la fijación de los precios de referencia (10-14), tanto de los reembolsos $(15,16)$ como de los sistemas de copagos diferenciales. De este modo se desalienta la compra de los productos más caros, debido a que es el paciente quien debe pagar la diferencia entre el precio de referencia y el precio del producto seleccionado. Es conveniente que los procedimientos de fijación de precios sean lo más simples y automáticos posibles para evitar juicios discrecionales de las autoridades regulatorias, que afectarían la viabilidad y el impacto de la regulación.

Otro instrumento para obtener mejores precios en los medicamentos priorizados es la implementación de compras centralizadas o la negociación con la industria, para lo cual se requiere una sólida capacidad técnica para verificar la información presentada por las empresas productoras. Al igual que con los cambios en las modalidades de contratación y adquisición, es necesario contar con gran capacidad técnica para adoptar y sostener un sistema basado en esquemas diferenciales de copago. De esta manera, el Estado ejerce una regulación desde la demanda, donde el factor más importante es la centralización y el aprovechamiento de las economías de escala.

El empleo de listas con revisión permanente es otra herramienta de racionalización y contención del gasto farmacéutico ampliamente utilizada en países europeos (17-20), Australia y Canadá. En particular, las listas positivas de medicamentos a ser financiados se utilizan de forma mayoritaria dentro de la Unión Europea y también en varios países de América Latina. La delimitación de las listas positivas constituye una herramienta básica en esos países para su política sanitaria, sea que se trate de la negociación de precios con la industria (Francia, Italia, Reino Unido, Suecia), la determinación de niveles de reembolso (España, Francia, Portugal), el armado de grupos terapéuticos o el establecimiento de precios de referencia (Alemania, España, Francia, Holanda, Italia, Portugal, Reino Unido y otros).

Los precios de referencia deben ser fijados para conjuntos homogéneos de medicamentos, donde su definición constituye una competencia técnica. Mientras que al definir precios de referencia para los reembolsos resulta relevante definir conjuntos homogéneos por clase terapéutica, al definir precios de referencia para el canal comercial puede resultar más conveniente hacerlo por principio activo.

La evaluación fármaco-económica se institucionalizó en varios países desarrollados (16). Primero fue Australia (21), luego la mayoría de los países de la Unión Europea (11), y actualmente, Estados Unidos busca incorporar una agencia de evaluación de tecnologías sanitarias que ayude a garantizar la eficiencia en el uso de los recursos destinados a la adquisición de medicamentos y a contener la expansión del gasto farmacéutico.

En América Latina, en los últimos años se observan importantes avances en el desarrollo de agencias para la evaluación económica, como es el caso de la creación de la Comisión Nacional de Incorporación de Tecnologías (CONITEC) en el Sistema Único de Salud de Brasil; el Centro Nacional de Excelencia Tecnológica en Salud (CENETEC), en México y, más recientemente, el Instituto de Evaluación Tecnológica en Salud (IETS), en Colombia, establecido a través de

CUADRO 2. Comparación de modelos de regulación económica de medicamentos en países seleccionados, varios años

\begin{tabular}{|c|c|c|c|}
\hline País & Método de regulación & Año & Institución con autoridad regulatoria \\
\hline Alemania & $\begin{array}{l}\text { Solo precios de referencia en el canal institucional } \\
\text { para genéricos y marcas comerciales de la misma } \\
\text { clase terapéutica }\end{array}$ & $\begin{array}{l}\text { Desde 1989; } \\
\text { se actualizan } \\
\text { anualmente }\end{array}$ & $\begin{array}{l}\text { Asociación Alemana de Fondos de Enfermedad. La } \\
\text { industria farmacéutica puede modificar los precios }\end{array}$ \\
\hline Australia & $\begin{array}{l}\text { El precio se fija en función de varios parámetros: } \\
\text { (1) Precios de marcas alternativas, (2) Costos de } \\
\text { producción (insumos) y (3) Volumen esperado de } \\
\text { prescripciones. } \\
\text { Se definen distintos precios para diferentes } \\
\text { indicaciones }\end{array}$ & $\begin{array}{l}\text { Desde 1998; } \\
\text { se actualizan } \\
\text { anualmente }\end{array}$ & Pharmaceutical Benefit Pricing Authority \\
\hline Brasil & $\begin{array}{l}\text { Regímenes diferenciados y un techo de precios } \\
\text { calculado con base en un índice de precios, un } \\
\text { factor de productividad y otro de ajuste de precios } \\
\text { relativos intra y entre sectores }\end{array}$ & 2003 & $\begin{array}{l}\text { Cámara de Regulación del Mercado } \\
\text { de Medicamentos (CMED), compuesta por los } \\
\text { ministerios de Salud, Hacienda, Justicia, Desarrollo y } \\
\text { Casa Civil (Ley No. } 10742 \text { [2003]) }\end{array}$ \\
\hline Canadá & $\begin{array}{l}\text { Regímenes diferenciados para productos protegidos } \\
\text { bajo patente y genéricos. No se regula el precio de } \\
\text { medicamentos sin patentes }\end{array}$ & 1987 & $\begin{array}{l}\text { Los precios de medicamentos son fijados por el } \\
\text { Patented Medicine Prices Review Board. Es una } \\
\text { organización independiente, un cuerpo cuasi-judicial }\end{array}$ \\
\hline Colombia & $\begin{array}{l}\text { Se establecen tres regímenes de regulación: libertad } \\
\text { vigilada, libertad regulada y control directo. Se } \\
\text { implementó un precio de referencia. Recientemente } \\
\text { se estableció que, por lo menos una vez al año, se } \\
\text { definirán los 'subprecios' del precio de referencia } \\
\text { para todos los medicamentos comercializados en } \\
\text { el país }\end{array}$ & 2011 & $\begin{array}{l}\text { La Comisión Nacional de Precios de Medicamentos } \\
\text { y Dispositivos Médicos, integrada por el Ministerio } \\
\text { de Comercio, Industria y Turismo, el Ministro de la } \\
\text { Protección Social y un delegado personal del Presidente } \\
\text { de la república (Ley No. } 100 \text { [1994] y Circular No. } 002 \\
\text { [2011] de la mencionada Comisión ) }\end{array}$ \\
\hline
\end{tabular}

(Continúa) 
CUADRO 2. (Continuación)

\begin{tabular}{|c|c|c|c|}
\hline País & Método de regulación & Año & Institución con autoridad regulatoria \\
\hline Ecuador & $\begin{array}{l}\text { Se definió un margen máximo de } 20 \% \text { de utilidad } \\
\text { para el fabricante o exportador, porcentaje calculado } \\
\text { con base en los costos y gastos declarados por } \\
\text { las empresas. En } 2011 \text { se incorporó un nuevo } \\
\text { marco regulatorio que distingue tres regímenes } \\
\text { de regulación: libertad vigilada, libertad regulada y } \\
\text { control directo (Decreto No. } 777 \text { ) }\end{array}$ & 2011 & $\begin{array}{l}\text { El Consejo Nacional de Fijación y Revisión de Precios } \\
\text { de Medicamentos de Uso Humano, integrado por el } \\
\text { Ministerio de Salud Pública, el Ministerio de Industrias } \\
\text { y Productividad, el Ministerio de Coordinación de } \\
\text { Desarrollo Social y el Ministerio Coordinador de la } \\
\text { Producción, Empleo y Competitividad (Resolución No. } \\
003 \text { [2011]) }\end{array}$ \\
\hline El Salvador & Sistema de precios de referencia, con base en los & 2012 & Dirección Nacional de Medicamentos \\
\hline
\end{tabular}
valores internacionales de referencia. En ningún caso el precio de los medicamentos podrá ser superior al promedio de venta en América Central

España Establecimiento de precios a partir de sus costos y el análisis caso por caso

2006 y 2012

Honduras Porcentaje máximo de utilidad bruta, determinado por la Secretaría de Industria y Comercio

Inglaterra

Límite en retorno a ganancias. El ajuste no se hace en forma individual sino en relación con las ganancias obtenidas por el laboratorio para su listado de medicamentos

México Se definen precios de referencia internacionales, pero los oferentes determinan su precio máximo de venta. Se propone el análisis de la estructura de costos definiendo costos de producción y márgenes de utilidad

Nicaragua Se fijan precios $\mathrm{CIF}^{\mathrm{a}}$ para medicamentos importados y se hacen comparaciones para los nacionales. También se fijan márgenes para minoristas y mayoristas, con porcentajes diferenciales para genéricos

Paraguay A través de coeficientes diferenciados, según correspondan a productos fabricados localmente, importados a granel o terminados. También se realizan acuerdos con el sector farmacéutico para bajar los precios

Sudáfrica Precios de referencia que eliminan reembolsos y descuentos. Se establece una tarifa para mayoristas y farmacéuticos.

Sistema transparente: los precios figuran en el envase de cada producto y en una página web de público acceso

Turquía Se utilizan precios de referencia tanto para los productos de marca como para los genéricos, con base en los de países europeos seleccionados. Luego se añade al precio de fábrica un $8 \%$ de impuesto al valor agregado más las ganancias del distribuidor y del farmacéutico

Venezuela Sistema de precios mixtos (regulados y libres). Solo se regulan los medicamentos esenciales. El Gobierno establece su precio a través de un sistema de márgenes de ganancia es de 2005
Desde 1957; el último acuerdo

2008

2009

2011

1996 Un Comité de Precios, cuyos miembros son designados por los ministros representantes de los departamentos de Salud, Finanzas y Justicia, la Comisión de Competencia Farmacéutica y los consumidores (sin presencia de la industria farmacéutica)

2007 Directorio General de Fármacos y Farmacias, que depende del Ministerio de Salud

2000
Corresponde al Consejo de Ministros establecer el régimen general de fijación de los precios industriales de los medicamentos financiados con cargo a fondos públicos, en tanto la Comisión Interministerial de Precios de Medicamentos debe fijar los precios industriales máximos de los mismos (Ley No. 29/2006; Real Decreto No. 200/2012)

Consejo Consultivo Multisectorial de Medicamentos (COMUDEM). Tiene un rol rector y está compuesto, entre otras entidades, por la Secretaría de Salud, el Colegio Químico Farmacéutico, el Instituto Hondureño de Seguridad Social, la Secretaría de Industria y Comercio y el Consejo de Defensa al Consumidor, la Asociación de Fabricantes de Productos Farmacéuticos y la Asociación de Propietarios de Farmacia (Acuerdo No. 606 del 27/3/2000,)

Pharmaceutical Price Regulation Scheme

Comisión Coordinadora para la Negociación de Precios de Medicamentos y Otros Insumos para la Salud, creada en 2008 e integrada por las Secretarías de Hacienda y Crédito Público, Economía y Salud, el Instituto Mexicano del Seguro Social e Instituto de Seguridad y Servicios Sociales para los Trabajadores del Estado. asesor permanente.

Dirección de Defensa al Consumidor, organismo descentralizado del Ministerio de Fomento, Industria y Comercio (Reglamentación Ley No. 182. Acuerdo ministerial 029-2009)

Dirección Nacional de Vigilancia Sanitaria del Ministerio de Salud Pública y Bienestar Social (Decreto No. 20996 [1998])
El Gobierno, a través del Ministerio de Finanzas Ligeras y Comercio y la Cartera de Salud (Ley No. 37003 [2000])

Fuente: elaboración de los autores con base en las referencias 2, 22 y 23.

a Siglas en inglés para "Costo, seguro y flete". 
la Ley No. 1438 (2011). En el cuadro 2 se muestran modelos de regulación económica de medicamentos ya puestos en marcha en algunos países de América Latina y otras regiones.

Entre América Latina y los países europeos hay claras diferencias en términos de regulación económica de medicamentos (24-27). En primer lugar, mientras en América Latina los países continuaron por el camino de la desregulación de los precios de medicamentos, los países europeos acudieron con frecuencia a medidas de control y regulación. En segundo lugar, los países de América Latina presentan un panorama de regulación económica de medicamentos mucho más heterogéneo que los países de Europa. Por lo tanto, en la región las medidas dirigidas a controlar los precios de medicamentos resultan más incipientes, heterogéneas y cambiantes, con modificaciones en períodos cortos de tiempo (cuadro 3).

Una tercera diferencia con Europa radica en el grado de cumplimiento de las regulaciones de medicamentos en América Latina, así como en la debilidad institucional de sus organismos para fiscalizar dicho cumplimiento. Algunos países de América Latina reaccionaron fortaleciendo el rol del Estado, tanto en la racionalización y el control del gasto, como en la regulación de los precios. El grado de efectividad de la regulación varía de país a país. Esta divergencia resulta de tres hechos importantes: i) articulación de la estrategia de control de precios con una política nacional de medicamentos; ii) método de regulación de los precios, y iii) composición y estabilidad institucional de la autoridad regulatoria.

Los países latinoamericanos que se incorporaron a la Organización para la Cooperación y el Desarrollo Económicos (OCDE) recorrieron trayectorias diferentes para desplegar la regulación económica de medicamentos. México, por ejemplo, optó por incorporar un mercado de medicamentos genéricos intercambiables e implementó una política de control de precios centrada en la referenciación internacional de precios. Chile, por su parte, avanzó desde la Fiscalía Nacional Económica en el control del proceso de formación de

CUADRO 3. Modelos de control de precios de los medicamentos vigentes en América Latina, 2008

\begin{tabular}{|c|c|}
\hline $\begin{array}{l}\text { Sistema de } \\
\text { precios }\end{array}$ & Mercados \\
\hline Libre & $\begin{array}{l}\text { Argentina, Bolivia, }{ }^{a} \text { Chile, Costa Rica, El Salvador, } \\
\text { Guatemala, Panamá, }{ }^{\mathrm{b}} \text { Perú, República Dominicana y } \\
\text { Uruguay }\end{array}$ \\
\hline Controlado & $\begin{array}{l}\text { Brasil, Ecuador, El Salvador, }{ }^{c} \text { Honduras, Nicaragua y } \\
\text { Paraguay }\end{array}$ \\
\hline Mixto & Colombia, México y Venezuela \\
\hline
\end{tabular}

Fuente: elaboración de los autores con base en la referencia 22 y la revisión de la normativa actualizada de cada país.

a Bolivia cuenta con una ley de responsabilidad social en el manejo de medicamentos y establecimientos farmacéuticos aprobada parcialmente. La misma establece la creación del SENAMET (Servicio Nacional de Medicamentos y Tecnología Médica-Sanitaria).

b Panamá estableció el control directo de precios entre 2001 y 2004.

c El Salvador sancionó su ley de medicamentos en marzo de 2012. precios para evitar prácticas anticompetitivas. Luego en 2011, desde el Instituto Nacional de Salud asumió una propuesta activa para consolidar un mercado de genéricos intercambiables de referencia. Sin embargo, aún no se logró progresar en lo que respecta al control de precios.

Brasil es el que más avanzó en la regulación económica de medicamentos dentro de la región (28-30). Por un lado, incorporó medidas centradas en la promoción de la oferta de genéricos intercambiables. Esto se logró mediante la reglamentación de requisitos de intercambiabilidad, la distinción de los envases de los productos genéricos y una activa producción pública. Por otro lado, implementó una política de regulación de precios que distingue regímenes de control de precios diferenciales para productos innovadores con ventajas terapéuticas, medicamentos "me too" ${ }^{4} \mathrm{y}$ genéricos.

\section{RECOMENDACIONES PARA PAÍSES DE AMÉRICA LATINA}

En base a lo expuesto, se recomiendan medidas tanto para contener el costo como el gasto en medicamentos, considerando los objetivos que persiguen las políticas y las herramientas posibles que se pueden desarrollar y adoptar. Respecto al primer eje, se identifican dos objetivos clave: i) garantizar la competitividad en el mercado y ii) garantizar precios asequibles. Las herramientas para el primero son el control de prácticas anticompetitivas y la promoción de genéricos, mientras que para el segundo objetivo lo son el control de precios y de los márgenes de comercialización y las negociaciones de precios. En particular, las recomendaciones de regulación económica para contener el costo de los medicamentos son: a) articular acciones entre autoridades sanitarias y de defensa de la competencia para promover la investigación del proceso de formación de precios, así como identificar y sancionar prácticas colusivas; b) incorporar estrategias de promoción del uso de medicamentos genéricos; c) fomentar la oferta de genéricos mediante el acortamiento de los tiempos para su salida al mercado y d) establecer reglamentaciones que obliguen tanto a prescriptores como a farmacéuticos a utilizar medicamentos genéricos.

Para el segundo eje también se identifican dos objetivos claves: i) contener el gasto público en medicamentos y ii) garantizar la eficiencia del gasto en medicamentos. En este caso, las herramientas para el primero son la promoción del uso de genéricos, cambios en las modalidades de contratación y adquisición, copagos y la fijación de precios para el reembolso, en tanto que para el segundo objetivo la herramienta principal es la evaluación económica de medicamentos y la financiación selectiva.

\footnotetext{
Los medicamentos "me too" son nuevas moléculas que no presentan ventajas terapéuticas sobre los medicamentos ya existentes, pero que se comercializan a precios más elevados en el mercado.
} 
Para este eje - la contención del gasto en medicamentos- las regulaciones económicas recomendadas son: a) incorporar los genéricos dentro de grupos para la determinación de precios de referencia y de reembolso; b) seleccionar los medicamentos de mayor costo para que sean incluidos en compras centralizadas por parte de las instituciones sanitarias; c) determinar los medicamentos de mayor beneficio terapéutico para confeccionar una lista ampliada de medicamentos esenciales cubiertos dentro de los planes o programas de salud obligatorios de cada país; d) regular los copagos para evitar que perjudiquen el acceso a productos esenciales; e) incorporar precios de referencia para los reembolsos de los seguros de salud, centrados en productos genéricos; f) priorizar los medicamentos de mayor beneficio terapéutico para que se establezcan sus precios de referencia; g) incluir criterios de beneficio terapéutico para la priorización de principios activos e identificación de las clasificaciones terapéuticas más relevantes; $h$ ) jerarquizar los medicamentos, de mayor a menor beneficio terapéutico, para realizar los análisis de costo-efectividad, e i) confeccionar listas negativas de medicamentos que no serán reembolsados, en función de criterios referidos al producto (valor médico y terapéutico, falta de alternativas, seguridad), a factores económicos (costo-efectividad, precios de referencia, previsiones de mercado) y a la patología de que se trate (severidad, cantidad de pacientes, necesidad).

\section{SYNOPSIS}

\section{Alternatives for adopting policies centered on access to medicines}

Latin America is adopting regulations that bear on medicinal costs and spending. The regulations have four main goals: i) to guarantee a competitive market, ii) to ensure affordability for individual consumers (commercial channel), iii) to contain public spending on medicines (institutional channel), and iv) to guarantee efficient spending on medicines. The experience of Latin America differs from that of countries in developed regions. In the latter, the countries tend to have similar policies, both in promoting generic medicines and in price control strategies, and in optimizing and containing pharmaceutical expense. In contrast, in Latin America, certain institutional weaknesses impede the consolidation and application of an effective regulatory policy. This paper reviews the experience gained through the adoption of economic regulations aimed at reducing spending and improving access to medicines, suggests lessons learned at the international level, and offers recommendations for the countries of Latin America. Its purpose is to offer key elements to decision-makers and the authorities of the countries concerned in pursuing economic regulation of medicines.

Keywords: Drug price; drugs, generic; financing, health; health investments; Latin America.

\section{REFERENCIAS}

1. Organización Panamericana de la Salud. Guía para la implementación de estrategias de medicamentos genéricos en los países de América Latina y el Caribe como mecanismo para mejorar el acceso a medicamentos; 2011. Disponible en: http://apps.who.int/medicined ocs/documents/s19196es/s19196es.pdf Acceso el 8 de noviembre de 2012.

2. Tobar F. Estrategias económicas y financiamiento del medicamento. En: Bermudez J, Oliveira MA, Esher A, eds. Acceso a Medicamentos: Derecho fundamental, papel del Estado. Río de Janeiro: ENSP; 2004.

3. Kanavos P, Vandoros S, Irwin R, Nicod E, Casson M, Medical Technology Research Group-LSE Health, et al. Differences in costs of and access to pharmaceutical products in the EU. Brussels: European Parliament; 2011. P. 92.

4. Vogler S. The impact of pharmaceutical pricing and reimbursement policies on generics uptake: implementation of policy options on generics in 29 european countries-an overview. Generics and Biosimilars Initiative Journal. 2012;1(2):93-100.

5. Puig-Junoy J. Políticas de fomento de la competencia en precios en el mercado de genéricos: lecciones de la experiencia europea. Gac Sanit. 2010;24(3):193-9.
6. Nguyen A, Kaplan WA, Laing R. Policy options for promoting the use of generic medicines in developing and transitional countries: a review paper. Geneva: World Health Organization; 2008.

7. Dylst P, Simoens S. Generic Medicine Pricing Policies in Europe: Current Status and Impact. Pharmaceuticals. 2010;3(3):471-81.

8. Pharmaceutical Pricing and Reimbursement Information (PPRI). Portugal Pharma Profile Vienna: European Commission, Health and Consumer Protection Directorate-General and the Austrian Ministry of Health, Family and Youth; 2008.

9. Garcia R. España prepara un nuevo paquete de medidas de contención del gasto en medicamentos, 2011. Disponible en: http://www.correofarmaceutico. com/2011/07/04/al-dia/profesion/ el-gobierno-prepara-mas-medidasde-contencion-para-el-interterrito rial Acceso el 16 de noviembre de 2012.

10. López-Casasnovas G, Puig-Junoy J. Review of the literature on reference pricing. Health Policy. 2000;54(2):87-123.

11. Vogler S, Habl C, Leopold C, RosianSchikuta I, Joncheere Kd, Thomsen TL. Pharmaceutical Pricing and Reimbursement Information (PPRI) Report. Vi- enna: European Commission, Health and Consumer Protection DirectorateGeneral and Austrian Federal Ministry of Health, Family And Youth; 2008.

12. Danzon PM, Ketcham JD. Reference Pricing of Pharmaceuticals for Medicare: Evidence from Germany, the Netherlands and New Zealand. Cambridge: National Bureau of Economic Research; 2004.

13. Yadav P. Differential Pricing for Pharmaceuticals. Review of current knowledge, new findings and ideas for action. Zaragoza: MIT-Zaragoza International Logistics Program; 2010.

14. Brekke KR, Königbauer I, Straume OR. Reference Pricing of Pharmaceuticals. J Health Econ. 2005;26(3):613-42.

15. Espín J, Rovira J. Analysis of Differences and Commonalities in Pricing and Reimbursement Systems in Europe. Brussels: DG Enterprise and Industry of the European Commission; 2007.

16. Drummond M, Jönsson B, Rutten F, Stargardt T. Reimbursement of pharmaceuticals: reference pricing versus health technology assessment. Eur J Health Econ. 2010;12(3):263-71.

17. Pharmaceutical Pricing and Reimbursement Information (PPRI). France Pharma Profile. Vienna: European Commission, Health and Consumer Protection Direc- 
torate-General and the Austrian Ministry of Health, Family and Youth; 2008.

18. Pharmaceutical Pricing and Reimbursement Information (PPRI). Germany Pharma Profile. Vienna: European Commission, Health and Consumer Protection Directorate-General and the Austrian Ministry of Health, Family and Youth; 2008.

19. Pharmaceutical Pricing and Reimbursement Information (PPRI). Italy Pharma Profile. Vienna: European Commission, Health and Consumer Protection Directorate-General and the Austrian Ministry of Health, Family and Youth; 2007.

20. Pharmaceutical Pricing and Reimbursement Information (PPRI). Sweden Pharma Profile. Vienna: European Commission, Health and Consumer Protection Directorate-General and the Austrian Ministry of Health, Family and Youth; 2007.

21. Birkett DJ, Mitchell AS, McManus P. A Cost-Effectiveness Approach to Drug
Subsidy and Pricing in Australia. Health Aff (Millwood). 2011;20(3):104-14.

22. Tobar F, Moretti C. Regulación económica de medicamentos en América Latina. Isalud. 2009;4(17):37-44.

23. Seiter A. A practical approach to pharmaceutical policy. Washington D.C: The World Bank; 2010.

24. Homedes N, Linares RL, Ugalde A. Generic Drug Policies in Latin America. Texas: World Bank; 2005.

25. Tobar F. Economía de los medicamentos genéricos en América Latina. Rev Panam Salud Publica. 2008;23(1):59-67.

26. Tobar F. Modelos de regulación económica de medicamentos en América Latina, 2008. Disponible en: http:// www.federicotobar.com.ar/nf_pdf2/ Regulacion.pdf Acceso el 16 de noviembre de 2012.

27. Tobar F, Sánchez D. El impacto de las políticas de medicamentos genéricos sobre el mercado de medicamentos en tres países del MERCOSUR. Montevideo: Fundación Carolina; 2005.

28. Maierovitch C. Política Vigente para Regulamentação de Medicamentos no Brasil. Brasilia: ANVISA; 2004.

29. Luiza VL, Castro CO, Marín N. Estudio de vigilancia de políticas farmacéuticas, Nivel 1 (estructura y procesos) realizado en países de Latinoamérica y el Caribe en 2003. Río de Janeiro: NAF/ENSP/ FIOCRUZ/OPS/WDC; 2006.

30. Bermúdez J, Possas C. Análisis crítico de la política de medicamentos en el Brasil. Boletín de la Oficina Sanitaria Panamericana. 1995;119:270-7.

Manuscrito recibido el 21 de diciembre de 2011. Aceptado para publicación, tras revisión, el 9 de septiembre de 2012. 